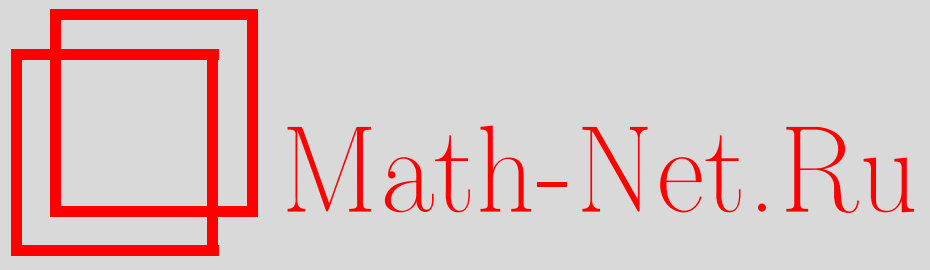

А. С. Холево, М. Е. Широков, Критерий слабой компактности для семейств обобщенных квантовых ансамблей и его следствия, Теория вероятн. и ее примен., 2015, том 60, выпуск 2, 402-408

DOI: https://doi.org/10.4213/tvp4627

Использование Общероссийского математического портала Math-Net.Ru подразумевает, что вы прочитали и согласны с пользовательским соглашением http://www . mathnet.ru/rus/agreement

Параметры загрузки:

IP: 54.80 .73 .141

26 апреля 2023 г., $17: 06: 01$

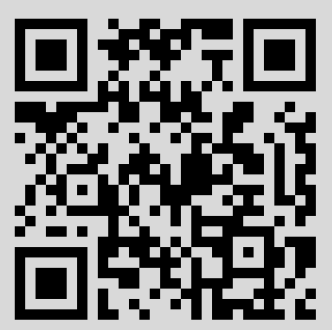


20. Prachar K. Primzahlverteilung. Berlin: Springer-Verlag, 1978, 421 p.

21. Виноградов И. М. Метод тригонометрических сумм в теории чисел. М.: Наука, 1980, $144 \mathrm{c}$.

22. Архипов Г.И., Караиуба А.А., Чубариков В.Н. Теория кратных тригонометрических сумм. М.: Наука, 1987, 368 с.

23. Стечкин С. Б. О средних значениях модуля тригонометрической суммы. - Тр. МИАН СССР, 1975, т. 134, с. 283-309.

24. Wooley T.D. Vinogradov's mean value theorem via efficient congruencing. - Ann. Math., 2012, v. 175, № 3, p. 1575-1627.

25. Parsell S. T., Prendiville S. M., Wooley T.D. Near-optimal mean value estimates for multidimensional Weyl sums. - Geom. Funct. Anal., 2013, v. 23, № 6, p. 1962-2024.

26. Linnik U. V. On Weyl's sums. - Матем. сб., 1943, т. 12, № 1, p. 28-39.

27. Линник Ю. В., Островский И. В. Разложения случайных величин и векторов. М.: Наука, 1972, 479 с.

28. Lukacs E., Laha R. G. Applications of characteristic functions. London: Griffin, 1964, $202 \mathrm{p}$.

29. Лукач E. Характеристические функции. М.: Наука, 1979, 423 с.

30. Shohat J. A., Tamarkin J.D. The Problem of Moments. Providence: Amer. Math. Soc., 1963, $144 \mathrm{p}$.

31. Ахиезер Н. И. Классическая проблема моментов и некоторые вопросы анализа, связанные с нею. М.: Физматгиз, 1961, 310 с.

32. Феллер В. Введение в теорию вероятностей и ее приложения, т. 2. М.: Мир, 1984, $752 \mathrm{c}$.

33. Стоянов Й. Контрпримеры в теории вероятностей. М.: Факториал, 1999, 288 с.

34. Математическая энциклопедия, т. 3. Под ред. И. М. Виноградова. М.: Советская энциклопедия, 1982, 1184 стб.

35. Ulyanov $V . V$. Nonuniform estimates of the density of the squared norm of a Gaussian vector in Hilbert space. - New Trends in Probability and Statistics, v. 1. Ed. by V. Sazonov and T. Shervashidze. Utrecht/Vilnius: VSP/Mokslas, 1991, p. 255-263.

36. Ulyanov $V$. V. On Gaussian measure of balls in $H$. - Frontiers in Pure and Applied Probability. II: Proceedings of the Fourth Russian-Finnish Symposium on Probability Theory and Mathematical Statistics. Ed. by A. N. Shiryaev et al. Moscow: TVP Science Publishers, 1996, p. 195-206.

37. Розовский Л. В. О гауссовой мере шаров в гильбертовом пространстве. - Теория вероятн. и ее примен., 2008, т. 53, в. 2, с. 382-390.

Поступила в редакцию 2.II. 2015

(c) 2015 г.

ХОЛЕВО А.С. ${ }^{*}$, ШИРОКОВ М. Е.*

\title{
КРИТЕРИЙ СЛАБОЙ КОМПАКТНОСТИ ДЛЯ СЕМЕЙСТВ ОБОБЩЕННЫХ КВАНТОВЫХ АНСАМБЛЕЙ И ЕГО СЛЕДСТВИЯ ${ }^{1}$
}

\begin{abstract}
Одним из центральных результатов теории вероятностных мер на метрических пространствах является теорема Ю.В. Прохорова о слабой компактности подмножеств вероятностных мер. В данной заметке с помощью этой теоремы получен критерий компактности для семейств обобщенных квантовых ансамблей - борелевских вероятностных мер на множестве квантовых состояний - и рассмотрены его приложения в квантовой теории информации.
\end{abstract}

* Математический институт им. В.А.Стеклова РАН, Москва, Россия; e-mail: holevo@mi.ras.ru; msh@mi.ras.ru

1) Исследование выполнено за счет гранта Российского научного фонда (проект № 14-21-00162). 
Ключевые слова и фразы: квантовое состояние, обобщенный квантовый ансамбль, барицентрическое отображение, квантовый канал.

В квантовой статистике ключевым является понятие квантового состояния, которое математически описывается оператором плотности - положительным ядерным оператором с единичным следом в гильбертовом пространстве, ассоциированном с квантовой системой. Такой оператор является некоммутативным аналогом вероятностной меры с конечным или счетным числом атомов.

Пусть $\mathscr{H}$ - сепарабельное гильбертово пространство, $\mathfrak{B}(\mathscr{H})$ - алгебра всех ограниченных операторов в $\mathscr{H}, \mathfrak{T}(\mathscr{H})$ - банахово пространство всех ядерных операторов со следовой нормой, т.е. класс Шаттена порядка $p=1$ (см. $[1,2])$. Выпуклое подмножество

$$
\mathfrak{S}(\mathscr{H})=\{\rho \in \mathfrak{T}(\mathscr{H}) \mid \rho \geqslant 0, \operatorname{Tr} \rho=1\}
$$

является полным сепарабельным метрическим пространством с метрикой, определяемой следовой нормой. Операторы из $\mathfrak{S}(\mathscr{H})$ называются квантовыми состояниями. Множество крайних точек $\operatorname{Ext} \mathfrak{S}(\mathscr{H})$ состоит из иистых состояний - одномерных проекторов в $\mathscr{H}$.

Энтропия фон Неймана состояния $\rho$ и квантовая относительная энтропия состояний $\rho$ и $\sigma$ определяются соответственно выражениями

$$
H(\rho)=-\sum_{i}\langle i|\rho \log \rho| i\rangle \quad \text { и } \quad H(\rho \| \sigma)=\sum_{i}\langle i|(\rho \log \rho-\rho \log \sigma)| i\rangle,
$$

в которых $\{|i\rangle\}$ - базис из собственных векторов состояния $\rho$, причем предполагается, что $H(\rho \| \sigma)=+\infty$, если носитель состояния $\rho$ (ортогональное дополнение ядра оператора $\rho$ ) не лежит внутри носителя состояния $\sigma$ [3, гл. 7].

Квантовым каналом называется линейное сохраняющее след вполне положительное отображение $\Phi: \mathfrak{T}\left(\mathscr{H}_{A}\right) \rightarrow \mathfrak{T}\left(\mathscr{H}_{B}\right)[3$, гл. 6]. Квантовые каналы являются некоммутативным аналогом марковских операторов в теории вероятностей; они играют роль динамических отображений в квантовой статистической механике и каналов связи в квантовой теории информации.

Конечный или счетный набор $\left\{\pi_{i}, \rho_{i}\right\}$ состояний $\rho_{i}$ с соответствующими вероятностями $\pi_{i}$ называется ансамблем, а состояние $\bar{\rho}=\sum_{i} \pi_{i} \rho_{i}-$ средним этого ансамбля. Квантовые ансамбли играют важную роль в квантовой теории информации, обусловленную, в частности, тем, что ряд характеристик квантового канала связи выражается в виде экстремальных значений энтропийных функционалов, определенных на множестве квантовых ансамблей. Например, $\chi$-пропускная способность ${ }^{2)}$ конечномерного квантового канала определяется выражением

$$
C_{\chi}(\Phi)=\sup _{\left\{\pi_{i}, \rho_{i}\right\}} \chi_{\Phi}\left(\left\{\pi_{i}, \rho_{i}\right\}\right),
$$

в котором супремум берется по всем ансамблям квантовых состояний, а

$$
\chi_{\Phi}\left(\left\{\pi_{i}, \rho_{i}\right\}\right) \doteq \sum_{i} \pi_{i} H\left(\Phi\left(\rho_{i}\right) \| \Phi(\bar{\rho})\right)=H(\Phi(\bar{\rho}))-\sum_{i} \pi_{i} H\left(\Phi\left(\rho_{i}\right)\right) .
$$

Нетрудно показать, что супремум в (1) можно брать только по подмножеству ансамблей, состоящих из не более чем $\left[\operatorname{dim} \mathscr{H}_{A}\right]^{2}$ состояний. Компактность этого подмножества (в топологии декартова произведения) позволяет показать достижимость супремума в (1), т.е. существование $\chi$-оптимальных ансамблей для канала $\Phi$, обладающих рядом замечательных свойств [4].

2) Эта величина, называемая в зарубежной литературе the Holevo capacity, тесно связана с пропускной способностью для передачи классической информации по квантовому каналу связи [3]. 
Важная особенность квантовых систем и каналов бесконечной размерности состоит в естественном появлении бесконечных (в общем случае «непрерывных») ансамблей, которые определяются как вероятностные меры на множестве всех квантовых состояний.

О п р е д е л е н и е 1 . Обобщенныл ансамблем квантовых состояний называется вероятностная борелевская мера $\mu$ на $\mathfrak{S}(\mathscr{H})$. Средним состоянием обобщенного ансамбля называется барицентр меры $\mu$, определяемый интегралом Бохнера

$$
\bar{\rho}(\mu)=\int_{\mathfrak{S}(\mathscr{H})} \rho \mu(d \rho) .
$$

Обычный ансамбль $\left\{\pi_{i}, \rho_{i}\right\}$ соответствует дискретной вероятностной мере $\sum_{i} \pi_{i} \delta\left(\rho_{i}\right)$, где $\delta(\rho)$ - дираковская мера, сосредоточенная в состоянии $\rho$.

Обозначим $\mathscr{P}(\mathscr{A})$ множество всех борелевских вероятностных мер на замкнутом подмножестве $\mathscr{A} \subseteq \mathfrak{S}(\mathscr{H})$, снабженное топологией слабой сходимости [5], [6]. Это множество является полным сепарабельным метрическим пространством [6, гл. II]. Заметим, что $\mathscr{P}(\mathscr{A})$ можно считать замкнутым подмножеством в пространстве $\mathscr{P}(\mathfrak{S}(\mathscr{H}))$.

Нетрудно показать, что барицентрическое отображение

$$
\mathscr{P}(\mathfrak{S}(\mathscr{H})) \ni \mu \mapsto \bar{\rho}(\mu) \in \mathfrak{S}(\mathscr{H})
$$

является непрерывной сюрьекцией (в соответствующих топологиях).

Необходимым и достаточным условием компактности метрического пространства $\mathscr{P}(\mathscr{A})$ является компактность подмножества $\mathscr{A}[5],[6]$. Это условие не выполнено в важных для приложений случаях, когда ${ }^{3)} \mathscr{A}=\mathfrak{S}(\mathscr{H}), \mathscr{A}=\operatorname{Ext} \mathfrak{S}(\mathscr{H})$ или $\mathscr{A}-$ множество всех сепарабельных состояний в тензорном произведении бесконечномерных гильбертовых пространств (см. ниже). Это обусловливает практическую значимость следующего результата, который опирается на теорему Ю. В. Прохорова о слабой компактности подмножеств вероятностных мер [5]-[7].

Теорема 1. Замкнутое подмножество $\mathscr{P}_{0} \subset \mathscr{P}(\mathfrak{S}(\mathscr{H})$ ) является компактньлм тогда и только тогда, когда его образ $\bar{\rho}\left(\mathscr{P}_{0}\right)$ при баричентрическом отображении (3) является компактным подмножеством в $\mathfrak{S}(\mathscr{H})$.

Поскольку компактность $\bar{\rho}\left(\mathscr{P}_{0}\right)$ непосредственно следует из компактности $\mathscr{P}_{0}$ в силу непрерывности отображения (3), нетривиальная часть теоремы 1 - это утверждение о компактности прообраза любого компактного подмножества в $\mathfrak{S}(\mathscr{H})$ при барицентрическом отображении (3). Можно показать, что это свойство отражает определенное соотношение между метрической и линейной структурами множества $\mathfrak{S}(\mathscr{H})$ (см. замечание в конце статьи).

Д о к а з а т е л ь с т в о т е о р е м ы 1 . Как отмечено выше, достаточно доказать компактность множества $\mathscr{P}_{0}$ при условии компактности его образа $\bar{\rho}\left(\mathscr{P}_{0}\right)$.

Пусть множество $\mathscr{A}=\bar{\rho}\left(\mathscr{P}_{0}\right)$ компактно. В силу общего критерия компактности для подмножеств множества $\mathfrak{S}(\mathscr{H})$ (см. [2]), для любого натурального числа $n$ существует проектор $P_{n}$ конечного ранга такой, что $\operatorname{Tr} \rho P_{n} \geqslant 1-n^{-3}$ для всех $\rho$ из $\mathscr{A}$. Без ограничения общности можно считать, что $\bigvee_{k=1}^{+\infty} P_{k}(\mathscr{H})=\mathscr{H}$, где $\bigvee$ обозначает замыкание линейной оболочки подпространств. Пусть $\widehat{P}_{n}$ - проектор на конечномерное подпространство $\bigvee_{k=1}^{n} P_{k}(\mathscr{H})$. Таким образом, $H=\sum_{n=1}^{+\infty} n\left(\widehat{P}_{n+1}-\widehat{P}_{n}\right)-$ самосопряженный оператор в $\mathscr{H}$, имеющий дискретный спектр конечной кратности и удовлетворяющий условию

$$
\operatorname{Tr} \rho H=\sum_{n=1}^{+\infty} n \operatorname{Tr} \rho\left(\widehat{P}_{n+1}-\widehat{P}_{n}\right) \leqslant \sum_{n=1}^{+\infty} n \operatorname{Tr} \rho\left(I_{\mathscr{H}}-\widehat{P}_{n}\right) \leqslant \sum_{n=1}^{+\infty} n^{-2}=h
$$

3) Здесь и далее считаем, что $\mathscr{H}$ - бесконечномерное пространство. 
для любого состояния $\rho$ из $\mathscr{A}$. Заметим, что величина $\operatorname{Tr} \rho H$ (конечная или бесконечная) определяется как предел неубывающей последовательности $\operatorname{Tr} \rho Q_{n} H$, где $Q_{n}-$ спектральный проектор оператора $H$, соответствующий отрезку $[0, n]$ (см. $[3$, гл. 10]).

Для произвольной меры $\mu \in \mathscr{P}_{0}$ имеем

$$
\int_{\mathfrak{S}(\mathscr{H})}(\operatorname{Tr} \rho H) \mu(d \rho)=\operatorname{Tr}\left(\int_{\mathfrak{S}(\mathscr{H})} \rho \mu(d \rho) H\right)=\operatorname{Tr} \bar{\rho}(\mu) H \leqslant h .
$$

Существование интеграла в левой части и первое равенство вытекают из теоремы о монотонной сходимости.

Пусть $\mathscr{K}_{\varepsilon}=\left\{\rho: \operatorname{Tr} \rho H \leqslant h \varepsilon^{-1}\right\}$. Множество $\mathscr{K}_{\varepsilon}$ компактно при всех $\varepsilon$ в силу леммы из [8]. Используя (4), для любой меры $\mu$ из $\mathscr{P}_{0}$ получаем

$$
\mu\left(\mathfrak{S}(\mathscr{H}) \backslash \mathscr{K}_{\varepsilon}\right)=\int_{\mathfrak{S}(\mathscr{H}) \backslash \mathscr{K}_{\varepsilon}} \mu(d \rho) \leqslant \varepsilon h^{-1} \int_{\mathfrak{S}(\mathscr{H}) \backslash \mathscr{K}_{\varepsilon}}(\operatorname{Tr} \rho H) \mu(d \rho) \leqslant \varepsilon,
$$

что доказывает плотность множества $\mathscr{P}_{0}$. Компактность этого множества следует теперь из теоремы Прохорова (см. [5]-[7]) и непрерывности отображения (3). Теорема доказана.

Критерий теоремы 1 позволяет доказать для некомпактного выпуклого множества $\mathfrak{S}(\mathscr{H})$ ряд свойств из теории выпуклых компактов (которые не имеют места для произвольных выпуклых ограниченных подмножеств метрических пространств).

Следствие 1. Выпуклое замыкание $e^{4)} \overline{\operatorname{conv}} \mathscr{A}$ любого замкнутого подмножества $\mathscr{A} \subseteq \mathfrak{S}(\mathscr{H})$ совпадает с множеством баричентров мер из $\mathscr{P}(\mathscr{A})$.

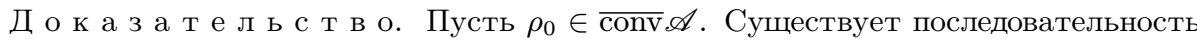
$\left\{\rho_{n}\right\}$, лежащая в выпуклой оболочке $\operatorname{conv} \mathscr{A}$ множества $\mathscr{A}$ и сходящаяся к $\rho_{0}$. Ясно, что каждое состояние $\rho_{n}$ является барицентром некоторой дискретной меры $\mu_{n} \in$ $\mathscr{P}(\mathscr{A})$. В силу теоремы 1 последовательность $\left\{\mu_{n}\right\}$ имеет частичный предел $\mu_{0} \in$ $\mathscr{P}(\mathscr{A})$. Из непрерывности отображения (3) следует, что $\rho_{0}-$ барицентр меры $\mu_{0}$. Следствие 1 доказано.

Заметим, что утверждение следствия 1 не выполняется для замкнутых ограниченных подмножеств положительного конуса класса Шаттена порядка $p>1$. Действительно, если $\mathscr{A}$ - пересечение единичной сферы и положительного конуса в таком пространстве, то $\overline{\operatorname{conv}} \mathscr{A}$ содержит нулевой оператор, который не может быть барицентром вероятностной меры с носителем в $\mathscr{A}$.

Важным приложением следствия 1 является интегральное представление любого сепарабельного состояния в тензорном произведении двух гильбертовых пространств. Пусть $\mathscr{A}-$ множество всех чистых состояний-произведений вида $\rho \otimes \sigma$ в $\mathfrak{S}(\mathscr{H} \otimes \mathscr{K})$. Состояние из $\mathfrak{S}(\mathscr{H} \otimes \mathscr{K})$ называется сепарабельнылм (несчепленным), если оно лежит в $\overline{c o n v} \mathscr{A}$. В силу следствия 1 всякое сепарабельное состояние можно представить как барицентр вероятностной меры на множестве чистых состояний-произведений, т.е. в виде «непрерывной» выпуклой комбинации чистых состояний-произведений. В [11] показано, что для некоторых сепарабельных состояний любая мера в таком представлении не имеет атомов, в частности, эти состояния нельзя представить в виде счетной выпуклой комбинации чистых состояний-произведений.

Важной областью применения критерия компактности в теореме 1 являются достаточные условия существования обобщенного $\chi$-оптимального ансамбля для бесконечномерного квантового канала. Как отмечено выше, для конечномерного канала всегда существуют $\chi$-оптимальные ансамбли, на которых достигается супремум в (1). При определении пропускных способностей бесконечномерного квантового канала $\Phi$

4) Выпуклое замыкание множества определяется как (топологическое) замыкание выпуклой оболочки этого множества [9], [10]. 
необходимо наложить некоторые ограничения на входные состояния, например, ограничение на среднюю энергию этих состояний. Зафиксируем положительный самосопряженный оператор $F$ в $\mathscr{H}_{A}$ и зададим линейные ограничения на входные состояния $\rho^{(n)}$ канала $\Phi^{\otimes n}$ вида

$$
\operatorname{Tr} \rho^{(n)} F^{(n)} \leqslant n E,
$$

где

$$
F^{(n)}=F \otimes \cdots \otimes I+\cdots+I \otimes \cdots \otimes F .
$$

Определим выходную $\chi$-величину обобщенного ансамбля $\mu \in \mathscr{P}\left(\mathfrak{S}_{(}\left(\mathscr{H}_{A}\right)\right)$ для канала $\Phi: \mathfrak{T}\left(\mathscr{H}_{A}\right) \rightarrow \mathfrak{T}\left(\mathscr{H}_{B}\right)$ выражением

$$
\chi_{\Phi}(\mu) \doteq \int_{\mathfrak{S}_{\left(\mathscr{H}_{A}\right)}} H(\Phi(\rho) \| \Phi(\bar{\rho}(\mu))) \mu(d \rho)=H(\Phi(\bar{\rho}(\mu)))-\int_{\mathfrak{S}_{\left(\mathscr{H}_{A}\right)}} H(\Phi(\rho)) \mu(d \rho),
$$

в котором вторая формула справедлива при условии $H(\Phi(\bar{\rho}(\mu)))<+\infty$ (и которое является интегральной версией выражения (2)). В силу теоремы кодирования для канала с линейными ограничениями (см. [8, предложение 3]), классическая пропускная способность канала $\Phi$ с ограничением (5) дается следующим регуляризованным выражением:

$$
C(\Phi, F, E)=\lim _{n \rightarrow+\infty} n^{-1} C_{\chi}\left(\Phi^{\otimes n}, F^{(n)}, n E\right),
$$

в котором оператор $F^{(n)}$ определен соотношением (6), а

$$
C_{\chi}(\Phi, F, E)=\sup _{\mu: \operatorname{Tr} \bar{\rho}(\mu) F \leqslant E} \chi_{\Phi}(\mu) .
$$

Обобщенным $\chi$-оптимальнылм ансамблем называется мера $\mu$, на которой достигается супремум в (7). Теорема 1 позволяет получить следующее достаточное условие существования таких ансамблей.

Теорема 2 (см. [12]). Если множество $\left\{\rho \in \mathfrak{S}\left(\mathscr{H}_{A}\right) \mid \operatorname{Tr} \rho F \leqslant E\right\}$ компактно, a выходная энтропия $H(\Phi(\rho))$ непрерывна на этом множестве, то существует обобщенный $\chi$-оптимальный ансамбль $\mu$ с носителем на множестве чистых состояний.

Условие теоремы 2 выполнено в важном для приложений случае, когда $\Phi-$ бозонный гауссовский канал, а $F-$ квадратичный оператор энергии (см. подробности в $[12])$.

Другая область применения критерия компактности в теореме 1 - исследование аналитических свойств различных функциональных конструкций, используемых в качестве характеристик квантовых систем и каналов. Одним из следствий теоремы 1 является следующий результат.

Следствие 2. Пусть $\mathscr{A}-$ замкнутое подмножество в $\mathfrak{S}(\mathscr{H})$, а $\mathscr{P}_{\rho}(\mathscr{A})-$ множество мер из $\mathscr{P}(\mathscr{A})$ с барицентром $\rho$. Если $f$ - полунепрерывная снизу, ограниченная снизу функиия на $\mathscr{A}$, то функиия

$$
f_{*}(\rho)=\inf _{\mu \in \mathscr{P}_{\rho}(\mathscr{A})} \int_{\mathscr{A}} f(\sigma) \mu(d \sigma)
$$

выпукла и полунепрерывна снизу на множестве $\overline{\operatorname{conv}} \mathscr{A}$. Для любого состояния $\rho$ из $\overline{\mathrm{conv}} \mathscr{A}$ инфимум в определении величины $f_{*}(\rho)$ достигается на некоторой мере из $\mathscr{P}_{\rho}(\mathscr{A})$.

Д о к а з а т е л ь с т в о. Корректность определения функции $f_{*}$ обеспечивается следствием 1 , а ее выпуклость проверяется непосредственно.

Нетрудно показать, что из полунепрерывности снизу и ограниченности снизу функции $f$ следует полунепрерывность снизу функционала

$$
\mathscr{P}(\mathscr{A}) \ni \mu \mapsto F(\mu)=\int_{\mathscr{A}} f(\sigma) \mu(d \sigma) .
$$


В силу теоремы 1 множество $\mathscr{P} \rho(\mathscr{A})$ компактно. Поэтому полунепрерывность снизу функционала (8) обеспечивает достижимость инфимума в определении величины $f_{*}(\rho)$ для каждого $\rho$ из $\overline{\operatorname{conv}} \mathscr{A}$.

Предположим, что функция $f_{*}$ не является полунепрерывной снизу. Тогда найдется такая последовательность $\left\{\rho_{n}\right\} \subset \overline{\operatorname{conv}} \mathscr{A}$, сходящаяся к состоянию $\rho_{0} \in \overline{\operatorname{conv}} \mathscr{A}$, что существует

$$
\lim _{n \rightarrow+\infty} f_{*}\left(\rho_{n}\right)<f_{*}\left(\rho_{0}\right) .
$$

Как показано выше, при каждом $n=1,2, \ldots$ в $\mathscr{P}_{\rho_{n}}(\mathscr{A})$ найдется мера $\mu_{n}$ такая, что $f_{*}\left(\rho_{n}\right)=F\left(\mu_{n}\right)$. В силу теоремы 1 существует подпоследовательность $\left\{\mu_{n_{k}}\right\}$, сходящаяся к некоторой мере $\mu_{0}$, которая, в силу непрерывности отображения (3), лежит в $\mathscr{P}_{\rho_{0}}(\mathscr{A})$. Из полунепрерывности снизу функционала (8) следует, что

$$
f_{*}\left(\rho_{0}\right) \leqslant F\left(\mu_{0}\right) \leqslant \liminf _{k \rightarrow+\infty} F\left(\mu_{n_{k}}\right)=\lim _{k \rightarrow+\infty} f_{*}\left(\rho_{n_{k}}\right),
$$

вопреки предположению (9). Следствие 2 доказано.

В случае $\mathscr{A}=\mathfrak{S}(\mathscr{H})$ следствие 2 дает аналитическое представление для выпуклого замыкания ${ }^{5)}$ полунепрерывной снизу ограниченной снизу, функции на $\mathfrak{S}(\mathscr{H})$, например для выпуклого замыкания выходной энтропии бесконечномерного квантового канала.

В случае $\mathscr{A}=\operatorname{Ext} \mathfrak{S}(\mathscr{H})$ следствие 2 позволяет определить бесконечномерный аналог конструкции «выпуклой крыши» (the convex roof construction), которая используется при построении различных характеристик смешанных квантовых состояний, в частности характеристик сцепленности [13]. Например, если $\mathscr{A}=\operatorname{Ext} \mathfrak{S}(\mathscr{H} \otimes \mathscr{K})$, а $f$ - энтропия частичного следа, то $f_{*}(\rho)$ - сцепленность формирования (entanglement of formation) состояния $\rho$ - является важной характеристикой состояния составной квантовой системы (см. подробности в [14]).

3 а м е ч а н и е. Как уже отмечалось, нетривиальная часть теоремы 1 - это утверждение о компактности прообраза любого компактного подмножества в $\mathfrak{S}(\mathscr{H})$ при барицентрическом отображении (3). Подробный анализ этого свойства (для ограниченных подмножеств полных сепарабельных метрических пространств) проведен в [15], где оно названо свойством $\mu$-компактности (оно заведомо выполнено для всех компактных подмножеств). Показано, что $\mu$-компактность отражает определенное соотношение между метрической и линейной структурами множества и что для подмножеств, обладающих этим свойством (и названных в [15] $\mu$-компактами), можно доказать ряд результатов из теории выпуклых компактов (так же, как это сделано в настоящей заметке и в [14] для $\mathfrak{S}(\mathscr{H})$ ). С помощью теоремы Ю. В. Прохорова получен критерий $\mu$-компактности и обнаружено, что, наряду с $\mathfrak{S}(\mathscr{H})$, этим свойством обладают множество вероятностных мер на любом полном сепарабельном метрическом пространстве с топологией слабой сходимости, множество квантовых каналов с топологией сильной сходимости и некоторые другие важные для приложений некомпактные подмножества полных сепарабельных метрических пространств.

\section{СПИСОК ЛИТЕРАТУРЫ}

1. Холево А. С. Вероятностные и статистические аспекты квантовой теории. М.Ижевск: Ин-т компьют. исслед., 2003, 404 с.

2. Сарымсаков T.А. Введение в квантовую теорию вероятностей. Ташкент: Фан, $1985,184 \mathrm{c}$.

5) Выпуклое замыкание функции - наибольшая полунепрерывная снизу выпуклая функция, не превосходящая данную функцию [9], [10]. 
3. Холево А. С. Квантовые системы, каналы, информация. М.: МЦНМО, 2010, 327 с.

4. Schumacher B., Westmoreland M.D. Optimal signal ensembles. - Phys. Rev. A., 2001, v. 63, № 2, p. 2308-2312.

5. Биллингсли П. Сходимость вероятностных мер. М.: Наука, 1977, 351 с.

6. Parthasarathy K.R. Probability Measures on Metric Spaces. New York-London: Academic Press, 1967, 276 p.

7. Прохоров Ю.В. Сходимость случайных процессов и предельные теоремы теории вероятностей. - Теория вероятн. и ее примен., 1956, т. 1, в. 2, с. 177-238.

8. Холево А. С. Классические пропускные способности квантового канала с ограничением на входе. - Теория вероят. и ее примен., 2003, т. 48, в. 2, с. 359-374.

9. Половинкин E. C., Балашов М. В. Элементы выпуклого и сильно выпуклого анализа. М.: Физматлит, 2004, 415 с.

10. Рокафеллар Р. Выпуклый анализ. М: Мир, 1973, 469 с.

11. Вернер Р. Ф., Холево А. С., Широков М. Е. О понятии сцепленности в гильбертовых пространствах. - Успехи матем. наук, 2005, т. 60, № 2, с. 153-154.

12. Холево A. C., Широков M. E. Непрерывные ансамбли и пропускная способность квантовых каналов бесконечной размерности. - Теория вероятн. и ее примен., 2005 , т. 50 , в. 1 , с. $98-114$.

13. Osborne T. J. Convex hulls of varieties and entanglement measures based on the roof construction. - Quantum Inform. Comput., 2007, v. 7, № 3, p. 209-227.

14. Широков M.E. Свойства пространства квантовых состояний и монотонные характеристики сцепленности. - Изв. РАН, 2010, т. 74, № 4, с. 189-224.

15. Протасов В.Ю., Широков М.Е. Обобщенная компактность в линейных пространствах и ее приложения. - Матем. сб., 2009, т. 200, № 5, с. 71-98.

Поступила в редакцию 21.I.2015

(c) 2015 г.

ЧИБИСОВ Д. М.*

\title{
ЗАКОН БОЛЬШИХ ЧИСЕЛ Я.БЕРНУЛЛИ И УСИЛЕННЫЙ ЗАКОН БОЛЬШИХ ЧИСЕЛ
}

\begin{abstract}
Обсуждается взаимосвязь между законом больших чисел и усиленным законом больших чисел для сумм независимых случайных величин. Сопоставляется математическое содержание этих законов и их связь с задачами математической статистики.
\end{abstract}

Ключевые слова и фразы: закон больших чисел, усиленный закон больших чисел, состоятельность оценок, сильная состоятельность оценок.

В докладе обсуждается взаимосвязь между законом больших чисел (з.б.ч.) и усиленным з.б.ч. (у.з.б.ч.) для сумм независимых случайных величин (эти законы выполняются и в ряде моделей зависимых величин, но цель доклада - сопоставить постановки задач и содержание этих результатов, и для этого достаточно ограничиться независимым случаем). Доклад носит методологический характер, он вызван тем, что, как показывает чтение статистической литературы, многие авторы при исследовании асимптотических свойств, скажем, оценок параметров считают нужным доказывать их сильную состоятельность, которая основана на у.з.б.ч. и которая по мнению автора не имеет отношения к статистическому содержанию.

Несмотря на их похожесть, з.б.ч. и у.з.б.ч. - принципиально разные утверждения, дающие ответы на разные вопросы. 3.б.ч. говорит, что относительная частота

* Математический институт им. В.А. Стеклова РАН, Москва, Россия; e-mail: chibisov@mi.ras.ru 